\title{
Effect of Aggressive Fluid Therapy on Outcomes after Endoscopic Retrograde Cholangiopancreatography: A Randomized Controlled Clinical Trial
}

\author{
Ramin Ghaderi ${ }^{1}$, Morteza Ghojazadeh ${ }^{2}$, Manouchehr Khoshbaten ${ }^{1, *}$, Amir Faravan ${ }^{3}$
}

1. Liver and Gastrointestinal Diseases Research Center, Tabriz University of Medical Sciences, Tabriz, Iran

2. Research Center of Evidence Based Medicine, Tabriz University of Medical Sciences, Tabriz, Iran

3. Department of Critical Care Nursing, Center for Nursing Care Research, Nursing and Midwifery Faculty, Iran University of Medical Sciences, Tehran, Iran

\section{* Corresponding Author:}

Manouchehr Khoshbaten, MD Imam Reza Hospital, Golgasht Ave., Tabriz, East Azerbaijan, Iran Tel: + 984133367499

Fax: + 985166614756

Email: khoshbatenm@tbzmed.ac.ir

Received: 27 Sep. 2018 Accepted: 07 Feb. 2019

\section{ABSTRACT}

\section{BACKGROUND}

Pancreatitis is a serious complication of endoscopic retrograde cholangiopancreatography (ERCP), which may lead to death. The purpose of this study was to evaluate the preventive effect of aggressive fluid therapy on the incidence of post-ERCP pancreatitis.

\section{METHODS}

In double-blind controlled condition, 240 patients were selected and divided into two groups. The treatment of the intervention group $(\mathrm{n}=120)$ included a dose of $20 \mathrm{~mL} / \mathrm{kg}$ of ringer lactate infusion within 90 minutes before ERCP and $3 \mathrm{~mL} / \mathrm{kg} / \mathrm{h}$ during ERCP followed by $3 \mathrm{~mL} / \mathrm{kg} / \mathrm{h}$ up to 8 hours. The treatment of the control group $(\mathrm{n}=120)$ included a dose of $1.5 \mathrm{~mL} / \mathrm{kg}$ of ringer lactate infusion during ERCP up to 8 hours later. Firstly, the patients were evaluated in terms of excessive fluid and serum amylase and pain level, and then they were re-evaluated 2, 8, and 24 hours after ERCP.

\section{RESULTS}

The mean age of the patients was $51.57 \pm 13.5$ years. Most of the patients were female (54.5\%). Pancreatitis was developed in 26 patients including $5.83 \%$ of the patients in the intervention group and $15.83 \%$ of the patients in the control group ( $p=0.013$ ). Pancreatic pain was seen in $7.5 \%$ of the patients in the intervention group and in $27.5 \%$ of the control group $(p<0.005)$. Hyperamylasemia was seen in $20.83 \%$ of the patients in the intervention group and in $35 \%$ of the control group $(p=0.014)$. The mean days of hospital admission was $1.308 \pm 0.807$ in the intervention group and $1.425 \pm 0.876$ in the control group ( $p=0.275)$.

\section{CONCLUSION}

Aggressive fluid therapy with ringer lactate solution before ERCP can effectively prevent postERCP pancreatitis, pancreatic pain, and hyperamylasemia.

\section{KEYWORDS:}

Endoscopic retrograde cholangiopancreatography, Pancreatitis, Hyperamylasemia, Ringer lactate, Clinical trial

Please cite this paper as:

Ghaderi R, Ghojazadeh M, Khoshbaten M, Faravan A. Effect of Aggressive Fluid Therapy on Outcomes after Endoscopic Retrograde Cholangiopancreatography: A Randomized Controlled Clinical Trial. Middle East J Dig Dis 2019;11:76-83. doi: 10.15171/mejdd.2018.131.

\section{INTRODUCTION}

Among the therapeutic and diagnostic endoscopic procedures used in gastroenterology, endoscopic retrograde cholangiopancreatography (ERCP) has the highest incidence of complications compared with other procedures. The incidence of post-ERCP complications including acute pancreatitis, bleeding, cholangitis, cholecystitis, and perforation is within the range of $4 \%$ to $16 \%$ 
and its mortality is about $1 \%$. The most common ERCP complication is post-ERCP acute pancreatitis with an incidence of over $15 \% .{ }^{1}$ Post ERCP pancreatitis (PEP) imposes a huge cost of prolonged hospital admission in such patients. ${ }^{2,3}$ The mechanisms that cause post-ERCP acute pancreatitis are not well known, but the pathogenic agents such as mechanical or thermal trauma of large duodenal papillae, high hydrostatic pressure secondary to the injection of contrast agent, and activation of pancreatic enzymes by releasing pre inflammatory cytokines that lead to bacterial infection are more likely to cause it. ${ }^{4}$

Regarding the risks of post-ERCP pancreatitis, various studies have been done on the ERCP techniques and pharmacological interventions to reduce the incidence of post-ERCP pancreatitis. Among the technical interventions, it is proved that prophylactic pancreatic stent placement can reduce the chance of developing pancreatitis in high risk individuals. ${ }^{5,6}$ However, in some cases due to the specific anatomy of the duct, guidewire cannulation and stent placement are difficult. The consequences of multiple attempts for stent placement are not desirable in the event of failure and subsequently increase the risk of post-ERCP pancreatitis. ${ }^{7}$ Pharmacological studies also include the use of octreotides, corticosteroids, protease inhibitors, and more than 35 different drugs in more than 100 clinical trials all of which are failed and only the use of rectal indomethacin in high-risk patients has been associated with reduced pancreatitis. ${ }^{8}$ Another method that has recently been considered is intensive periprocedural fluid resuscitation, which can inhibit pancreatic inflammatory response by maintaining pancreatic microcirculation. Relative dehydrated status in patients with ERCP exacerbates microcirculation disorder. The level of blood urea, which measures the hydration status, increases after ERCP, which is associated with the PEP expansion. ${ }^{9,10}$

Regardless of the etiology, hydration and aggressive intravenous fluid resuscitation (IVFR) are the main treatment of acute pancreatitis. ${ }^{11}$ Studies on animal models have introduced the small vessel hypoperfusion theory as the etiology of pancreatitis, and inflammation has been more severe in areas of tissue with hypoperfusion. ${ }^{12,13}$ Clinical studies in patients with acute pancreatitis have also shown that fluid therapy reduces the chance of pancreatic necrosis and bad prognosis. ${ }^{14,15}$ Fluid therapy also improves the results in patients with hypovolemic shock and improves pancreatic microvascular perfusion. ${ }^{16}$ The same evidence has led to the suggestion of a preventive treatment as pre-ERCP fluid therapy (to reduce the chance of pancreatitis). This method in case of being efficient will have less cost for the patient and the healthcare system and low side effects. The inadequacy of most drug regimens and the simplicity and availability of such a treatment have led to more attention to such methods in recent years but so far few studies have been conducted on this method and there is no clear answer in this field. Therefore, this study was conducted to investigate the short-term effect of aggressive fluid therapy with lactated ringer solution before ERCP on its outcomes.

The reason for the selection of lactated ringer solution was the existence of evidence on its role in reducing the systemic inflammatory response syndrome (as compared with normal saline), which may be due to a lower likelihood of metabolic acidosis (hyperchloremic). Moreover, anti-inflammatory properties have been reported for lactate. ${ }^{17}$

\section{MATERIALS AND METHODS}

\section{Study design and participants}

This study was a double-blind clinical trial performed on patients referring to the Endoscopy Department of Imam Reza Hospital for performing ERCP in Tabriz in 2016. The inclusion criteria were the indication of ERCP (choledocholithiasis, bile duct leak, and biliary obstruction) and willingness to participate in the study. Also the patients were excluded in the event of active cholangitis, sepsis, pregnancy, age over 70 years, chronic pancreatitis, active pancreatitis from gallstone, risk of fluid overload (Class II and above cardiac failure, renal failure diagnosed by creatinine clearance less than 40 $\mathrm{mL} / \mathrm{min}$, liver failure, respiratory failure as $\mathrm{O}_{2}$ sat $<90 \%$ at room conditions), peripheral edema, pulmonary edema, electrolyte imbalances such as $\mathrm{Na}>150 \mathrm{mEq} / \mathrm{L}$ or $\mathrm{Na}<130 \mathrm{mEq} / \mathrm{L}$ and previous history of ERCP associated with sphincterotomy (due to lower risk of pancreatitis). The blindness of the study was that the patients under the study and the researchers did not know the patients' study group.

Institutional Review Board of Tabriz University of Medical Sciences has approved the study protocol based 




Fig.1: CONSORT diagram of patients flow through the trial

on the principles of Helsinki Declaration. This trial has been registered in the Iranian Clinical Trials Registry (IRCT201608101213N4).

Each patient received a complete explanation about the ongoing standard of care, potential risks, and advantages of treatment, and then informed consents were obtained. The patients underwent a complete physical examination and measuring of oxygen saturation so that patients with upper extremity edema, ascites, and pulmonary rales in particular can be excluded.

Firstly the patients were evaluated in terms of excessive fluid and serum amylase and pain level. The patients were re-evaluated after ERCP and 2, 8, and $24 \mathrm{~h}$ later. Both patients and gastroenterologist evaluators were blinded to the type of the patients' hydration. This was not kept up during the follow-up period due to the nature of the investigation. The intervention group received fluid for 24 hours, while the control group did not receive. Paraclinical studies were performed on the basis of clinical indications among cases (Ultrasonography, CT scan, and abdominal radiography).

\section{Sample size and sampling method}

Since in this study the incidence of post ERCP pancreatitis was considered as a primary outcome and according to the published studies and Texts the incidence of pancreatitis was reported as $13 \%$, with $\alpha=0.05$, power of $80 \%$ and a difference of $10 \%$, the sample size was calculated as 240 and the participants were randomly assigned to two groups of $n=120$.

\section{Randomization}

After collecting the written consents, a number was given to each selected patient in a continuous order. A schedule was prepared in advance by RandList and the patients were assigned to the intervention or control groups based on their randomly assigned numbers. CONSORT diagram of patients flow through the trial shown in figure 1 . 


\section{Intervention}

The qualified individuals were randomly divided into the intervention and control groups before ERCP. For the intervention group (aggressive fluid therapy), a dose of $20 \mathrm{~mL} / \mathrm{kg} / \mathrm{h}$ of lactated ringer solution was given within 90 minutes before ERCP and $3 \mathrm{~mL} / \mathrm{kg} / \mathrm{h}$ was prescribed during ERCP, which lasted up to 8 hours. Then, it was reduced to $1.5 \mathrm{~mL} / \mathrm{kg} / \mathrm{h}$ if they did not have pain. For the control group, $1.5 \mathrm{~mL} / \mathrm{kg} / \mathrm{h}$ of lactated ringer solution was given during the ERCP, which lasted up to 8 hours after ERCP. Receiving intravenous fluid was discontinued when the participants were able to tolerate normal diet in both groups. Then, ERCP was performed for all patients using a guide wire. After completing ERCP, all patients were examined 2, 8, and 24 hours later and the study variables were reviewed and recorded. The examination was made in terms of fluid overload through clinical examinations such as edema in the ankles, pulmonary rales, or $\mathrm{O}_{2}$ saturation reduction.

\section{Outcome}

Post-ERCP pancreatitis within $24 \mathrm{~h}$ follow-up period was considered as the studied variable in this investigation. Post-ERCP pancreatitis has been defined as the presence of the two criteria: epigastric pain that radiates into the back with a score of more than 3 on the visual analog scale (VAS) and lasts until the next 24 hours, and hyperamylasemia defined as amylase level more than 3 times the normal $300 \mathrm{U} / \mathrm{L}$.

\section{Statistical analysis}

In order to evaluate the difference between student's $\mathrm{t}$ test to assess differences in means (age, level of pain, amylase, liver enzymes, bilirubin, creatinine, hematocrit, and hospitalization days) and qualitative variables (sex, pancreatic pain, and hyperamylasemia) the Chi-square test was used. A two-way repeated measurement analysis of variance (time-treatment interaction) was performed as well. The type of hydration (an inter-subject factor (group)) and time of measurements (as an in-subject factor (time)) were assessed in pain and amylase parameters. Descriptive information was presented through mean \pm standard deviation and the significance level was considered at level 0.05. SPSS software (version 21, SPSS Inc., Chicago, IL) was used to analyze the data.

\section{RESULTS}

Of 260 screened patients, 240 cases were finally included in the study and were classified into two groups of 120.The mean age of the patients was $51.57 \pm 13.5$ years (range: 14-87). 131 (54.5\%) patients were women. Table 1 shows the participants' profile and the results of the comparison between the target and control groups. As shown in the table, patients in both groups were matched according to age and sex distribution.

Sphincterotomy was performed in all patients. Balloon dilatation was performed in $109(98.83 \%)$ patients in the intervention group and $94(78 \%)$ patients in the control group ( $p=0.038)$.

Stone extraction baskets were used in $108(90 \%)$ patients in the intervention and $99(82.5 \%)$ patients in the control groups $(p=0.092)$. Metal stent was applied in two $(1.66 \%)$ patients in the intervention and $9(7.5 \%)$ patients in the control group $(p=0.031)$. The plastic stent was used in $10(8.33 \%)$ patients in the intervention and 16 $(13.33 \%)$ patients in the control groups $(p=0.213)$. Due to cannulation problems and the duration of cannulation, seven patients were excluded from the study due to the risk of post-ERCP pancreatitis. In addition to pancreatitis, which was the main outcome, ERCP complications occurred in five patients (residual stone in the tract, perforation, stuck in the basket). Major complications in patients under study are shown in Table 2.

Out of the 240 patients, pancreatitis occurred in 26 patients $(10.83 \%)$. Their mean age was $52.92 \pm 12.89$ years. In the intervention group receiving an aggressive fluid therapy, seven patients $(5.83 \%)$ had post ERCP pancreatitis. The mean age of these patients was $45 \pm$ 18.76 years. However, in the standard fluid therapy group, 19 (15.83\%) patients suffered from pancreatitis, which was significantly higher than the intervention group ( $p=0.013)$. The mean age of these individuals was $55.84 \pm 8.95$ years.

In the group receiving aggressive fluid therapy, pancreatic pain (score 3 or higher based on VAS) was reported by nine patients $(7.5 \%)$, which was significantly less than the control group $(p<0.005) .33$ patients in the control group $(27.5 \%)$ reported the 3 rd level pain or higher in at least one of the measurements. Figure 2 shows how the pain varies in three measurements. In the repeated 
Table 1: Basic characteristics of the patients under endoscopic retrograde cholangiopancreatography

\begin{tabular}{|c|c|c|c|}
\hline Variables & Aggressive hydration & Standard care & $P$ \\
\hline Male/female (n) & $58-62$ & $53-63$ & $0.517 *$ \\
\hline Normal bilirubin $(\leq 1 \mathrm{mg} / \mathrm{dL})$ & $51(42.5 \%)$ & $41(40.83 \%)$ & $0.552 *$ \\
\hline Young age $(<30 \mathrm{y})$ & $15(12.5 \%)$ & $7(5.83 \%)$ & $0.074 *$ \\
\hline Sphincterotomy & $120(100 \%)$ & $120(100 \%)$ & - \\
\hline Using ballon dilatation & $109(98.83 \%)$ & $94(78 \%)$ & $0.038 *$ \\
\hline Pancreatic duct injection & $24(20 \%)$ & $22(18.33 \%)$ & $0.74 *$ \\
\hline $\mathrm{Age}^{* * *}$ (year) & $50.28 \pm 15.07$ & $52.28 \pm 12.42$ & $0.220 * *$ \\
\hline Indication: Bile duct stone (\%) & $44.58 \dagger$ & $40.83 \dagger$ & - \\
\hline Bilirubin (total) (mg/dL) & $3.42(4.64)$ & $5.2(6.62)$ & $0.519 * *$ \\
\hline Bilirubin (direct) & $21.16(3.20)$ & $3.47(4.79)$ & $0.520 * *$ \\
\hline Blood Urea Nitrogen (mg/dL) & $30.75(11.82)$ & $33.48(14.89)$ & $0.120 * *$ \\
\hline Hemoglobin (mg/dL) & $12.39(2.02)$ & $12.14(1.95)$ & $0.366^{* *}$ \\
\hline Hematocrit (\%) & $37.31(6.57)$ & $37 / 04(5.01)$ & $0.305^{* *}$ \\
\hline Creatinine (mg/dL) & $0.91(0.25)$ & $0.97(0.45)$ & $0.312 * *$ \\
\hline Aspartate aminotransferase (IU/L) & $101.56(174.45)$ & $87.65(81.93)$ & $0.796 * *$ \\
\hline Alanine aminotransferase (IU/L) & $123.90(175.11)$ & $106.83(118.18)$ & $0.845^{* *}$ \\
\hline Alkaline phosphatase (IU/L) & $680.34(532.03)$ & $731.02(570.87)$ & $0.897 * *$ \\
\hline Days of hospital admission & $1.308 \pm 0.807$ & $1.425 \pm 0.876$ & $0.275 * *$ \\
\hline
\end{tabular}

Table 2: Results in the Study Groups

\begin{tabular}{|c|c|c|c|}
\hline Parameter & $\begin{array}{c}\text { Aggressive hydration }(n=120) \\
\text { N }(\%)\end{array}$ & $\begin{array}{c}\text { Standard care }(n=120) \\
\text { N }(\%)\end{array}$ & $P$ \\
\hline Post-ERCP pancreatitis & $7(5.83)$ & $19(15.83)$ & $\mathrm{p}=0.013$ \\
\hline Hyperamylasemia & $25(20.83)$ & $42(35)$ & $\mathrm{p}=0.014$ \\
\hline Pancreatic pain & $9(7.5)$ & $33(27.5)$ & $\mathrm{p}<0.005$ \\
\hline
\end{tabular}

measure analysis, the reported pain in both groups had a reducing trend $(p<0.005)$. Also, the effect of the intervention was significant $(p<0.005)$. The mean days of hospital admission was $1.308 \pm 0.807$ in the intervention group and $1.425 \pm 0.876$ in the control group ( $p=0.275$ ).

In the group receiving aggressive fluid therapy, hyperamylasemia was observed in at least one of the measurements in 25 patients $(20.83 \%)$ while this level was significantly higher in the control group $(p=0.014)$, and $42(35 \%)$ patients in the control group had hyperamylasemia in at least one of the measurements. Changes in serum amylase levels are shown in figure 3. The changes in the serum amylase level were not significant in the analysis by repeated measure in the two groups $(p=0.174)$. On the other hand, the effect of the intervention was reported as significant $(p<0.005)$. As seen in the figure, the level of amylase was lower in the group receiving aggressive fluid therapy.

\section{DISCUSSION}

This study was conducted to investigate the shortterm effect of pre-ERCP aggressive fluid therapy with ringer lactate solution on the post-ERCP outcomes in 240 patients, which showed that this method can reduce the incidence of pancreatitis, hyperamylasemia, and pain without serious complications. Based on clinical evidence, receiving intravenous fluid is the main intervention for the treatment of acute pancreatitis and is underlined by clinical guidelines. ${ }^{18,19}$ The positive effect of infusing enough fluid is probably caused by improved 


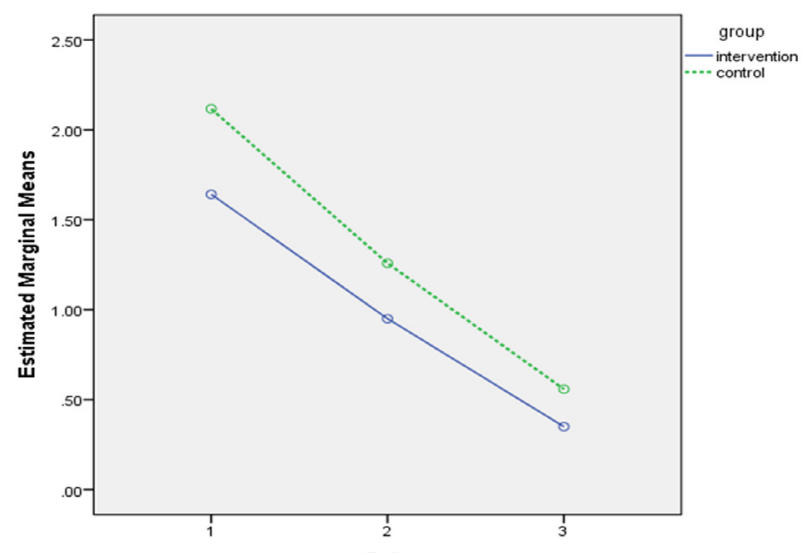

Fig.2: Mean level of pain reported by patients in 2,8 , and $24 \mathrm{~h}$ post endoscopic retrograde cholangiopancreatography

blood supply to tissues. Studies have shown that there is a significant relationship between post-ERCP pancreatitis risk and higher levels of blood urea nitrogen, which reflects fluid deficiency and dehydration. ${ }^{10}$

Clinical trials have shown that other markers of fluid deficiency (creatinine and high hematocrit) are associated with a possibility of organ failure. ${ }^{20,21}$ Therefore, with the supply of fluid, blood reperfusion is likely to improve and there is a possibility of preventing this process and its consequences. In addition, principally, patients should be fast before ERCP and this may take longer than expected and these conditions may aggravate the patients' dehydration. ${ }^{10}$ Despite all these evidence, the preventive effect of fluid therapy on pancreatitis has not yet been determined. An aggressive fluid therapy, even if it does not prevent post- ERCP pancreatitis, provides an opportunity to start a basic treatment even before the physician's diagnosis and could possibly reduce the severity of pancreatitis.

Among the studies that examined the effect of aggressive fluid therapy on the prevention of post-ERCP pancreatitis, there is a pilot study by Buxbaum and colleagues. ${ }^{17}$ In this study, 39 patients received aggressive fluid therapy with ringer lactate and 23 patients were considered as the control group receiving routine fluid (ringer lactate species). Patients in this study, which our present study is based on, were referred to an ERCP level 3 referral center, and exceeded fluid risk factors were considered as exclusion criteria. The results of this study showed that $17 \%$ of the patients in the control group

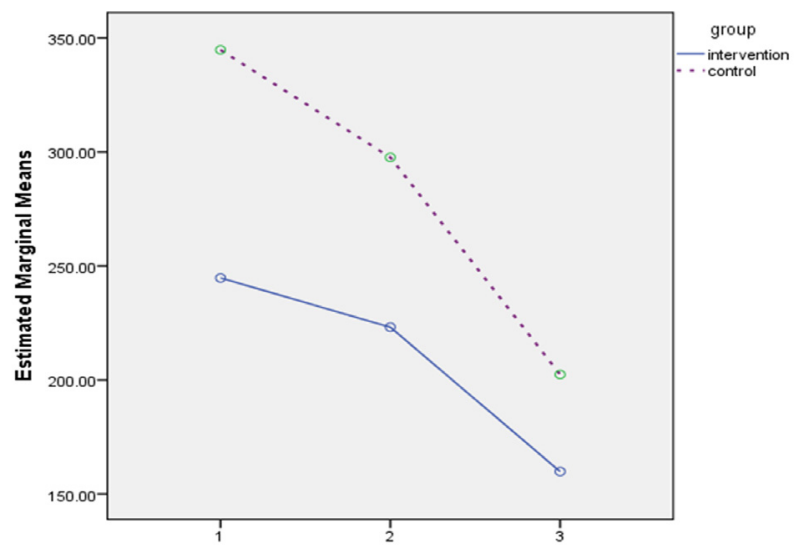

Fig.3: Mean level of amylase as measured in 2, 8, and 24 $h$ post endoscopic retrograde cholangiopancreatography in the participants

suffered from pancreatitis while post-ERCP pancreatitis did not occur in any of the patients with aggressive fluid therapy ( $p=0.016$ ). This difference was statistically significant, which is consistent with the results of our study. The difference in the incidence between these two studies is more likely to be attributed to the greater number of samples in our study that have increased the prediction power of the study and enclosed the sample to the clinical population. Contrary to the present study, aggressive fluid therapy had no significant effect on hyperamylasemia and pain in the study by Buxbaum and colleagues. In this study, hyperamylasemia was seen in $23 \%$ of the patients with aggressive fluid therapy and $39 \%$ in the control group, which was not statistically significant $(p=0.116)$. In the present study, in addition to the analysis of hyperamylasemia as a qualitative variable (presence or absence of hyperamylasemia), the level of amylase was analyzed as a quantitative variable and it was shown that the intervention had a significant effect on the amylase level of the patients. Amylase levels were fluctuating in both groups and its changes in two groups were not statistically significant, but the level of amylase had a more specific pattern in the intervention group and had a generally decreasing trend. Another difference between the two studies was the effect on pain. Epigastric pain was seen in $8 \%$ of the patients with aggressive fluid therapy and $22 \%$ in the control group, which was not significant unlike the present study $(p=0.146)$. This is why the present study has more samples and pancreatic pain was significantly lower in the aggressive fluid therapy 
group. Moreover, the amount of pain was analyzed as a quantitative variable and although the amount of pain was decreasing in both intervention and control groups, the effect of intervention was significant and the aggressive fluid therapy could reduce the pain. In addition to the superiority in terms of sample size, another advantage of this study was double-blind intervention. In the Buxbaum's study, the researchers were not blind to the patients' group. This point was considered in this study with regard to the point that the group was not obvious in the measurements and examination before, immediately after, and within 2 and 8 hours after ERCP. However, at $24 \mathrm{~h}$ only the intervention group received the fluid therapy, and the fluid therapy of the control group had been discontinued. Thus the patients group could be determined.

The analysis of the quantitative variables was another difference between the two studies, which allowed the examination in terms of these two variables. A recent study by Shayegan Nejad and his colleagues is another example in this regard. ${ }^{22}$ Their results in terms of the effect of aggressive fluid therapy on the incidence of post-ERCP pancreatitis, hyperamylasemia, and pain are consistent with this study. The advantage of this study over the study by Shayegan Nejad and colleagues is the higher sample size. Their sample size was 150 while it is 240 in our study. Therefore, the generalization power of this study to the community is greater in comparison with the previous studies. Another difference between this study and Shayegan Nejad's study is the time of the intervention. The onset of fluid therapy in this study was before the ERCP while fluid therapy was started with ERCP in Shayegan Nejad's study. The incidence of pancreatitis (as the main study variable) was $2.9 \%$ in this study ( $p=0.013$ ) while it was reported as $5.3 \%$ in Shayegan Nejad's study $(p=0.002)$. The results of these two studies promise a useful strategy to prevent the occurrence of post-ERCP pancreatitis. However, this preventive method has some limitations, and more studies are needed to confirm its details.

An aggressive fluid therapy may be hazardous to the elderly. Furthermore, this method may be more risky in cases with sodium retention (such as congestive heart failure, and chronic heart disease). Therefore, the benefits of this approach are limited among such patients. In ad- dition, the minimum amount of fluid that prevents pancreatitis is not yet known and a clinical trial is needed to determine an evidence-based method.

\section{Limitations}

This study has some limitations. Considering that studies on the efficacy of aggressive fluid therapy in preventing post-ERCP pancreatitis are currently at their early stages, the severity of pancreatitis was not included in the design of this study. It is suggested that subsequent studies address the effect of aggressive fluid therapy on the severity of pancreatitis by selecting a larger number of patients.

Exact exclusion criteria were used to minimize the adverse effects of aggressive fluid therapy; therefore, the outcomes cannot be attributed to all patients and further investigations on the side effects of fluid intake should be considered.

\section{CONCLUSION}

Pre-ERCP aggressive fluid therapy with ringer lactate solution can effectively prevent pancreatitis, pancreatic pain, and hyperamylasemia. This method did not cause complications for the patients who were not high risk to excessive fluid therapy.

\section{ETHICAL APPROVAL}

There is nothing to be declared.

\section{CONFLICT OF INTEREST}

The authors declare no conflict of interest related to this work.

\section{REFERENCES}

1. ASGE Standards of Practice Committee, Anderson MA, Fisher L, Jain R, Evans JA, Appalaneni V, et al. Complications of ercp. Gastrointest Endosc 2012;75:467-73. doi:10.1016/j.gie.2011.07.010

2. Arata S, Takada T, Hirata K, Yoshida M, Mayumi T, Hirota M, et al. Post-ERCP pancreatitis. J Hepatobiliary Pancreat Sci 2010;17:70-8. doi:10.1007/s00534-009-0220-5.

3. Gross V, Leser H, Heinisch A, Schölmerich J. Inflammatory mediators and cytokines--new aspects of the pathophysiology and assessment of severity of acute pancreatitis? Hepatogastroenterology 1993;40:522-30.

4. Rochester J. Risks of endoscopic retrograde cholangio- 
pancreatography and sphincterotomy. Tech Gastrointest Endosc 2008;10:14-21. doi:10.1016/j.tgie.2007.08.006.

5. Freeman ML. Role of pancreatic stents in prevention of post-ERCP pancreatitis. JOP 2004;5:322-7.

6. Arain MA, Freeman ML. Pancreatic stent placement remains a cornerstone of prevention of post-ercp pancreatitis, but it requires specialized techniques. Gastrointest Endosc 2015;81:156-8. doi:10.1016/j.gie.2014.09.020.

7. Choksi NS, Fogel EL, Cote GA, Romagnuolo J, Elta GH, Scheiman JM, et al. The risk of post-ercp pancreatitis and the protective effect of rectal indomethacin in cases of attempted but unsuccessful prophylactic pancreatic stent placement. Gastrointest Endosc 2015;81:150-5. doi: 10.1016/j.gie.2014.07.033.

8. Sherman S, Lehman G, Earle D, Watkins J, Freeman M, Parker H, et al. Does prophylactic steroid administration reduce the frequency and severity of post-ecrp pancreatitis? Randomized prospective multicenter study. Gastrointest Endosc 1996;43:320. doi:10.1016/S00165107(96)80121-0.

9. Kushner T, Majd N, Sigel K, Liverant ML, Wong SY, Patel KK, et al. 449 blood urea nitrogen as a predictor of development of post-endoscopic retrograde cholangiopancreatography pancreatitis: A case-control study. Gastrointest Endosc 2012;75:AB141-AB142.

10. Cote GA, Sagi SV, Schmidt SE, Lehman GA, McHenry L, Fogel E, et al. Early measures of hemoconcentration and inflammation are predictive of prolonged hospitalization from post- endoscopic retrograde cholangiopancreatography pancreatitis. Pancreas 2013;42:850-4. doi: 10.1097/MPA.0b013e318287c9d4.

11. Elmunzer BJ, Scheiman JM, Lehman GA, Chak A, Mosler P, Higgins PD, et al. A randomized trial of rectal indomethacin to prevent post-ERCP pancreatitis. $N$ Engl J Med 2012;366:1414-22. doi:10.1056/NEJMoa1111103.

12. Schmidt J, Ebeling D, Ryschich E, Werner J, Gebhard MM, Klar E. Pancreatic capillary blood flow in an improved model of necrotizing pancreatitis in the rat. J Surg Res 2002;106:335-41. doi:10.1006/jsre.2002.6464.

13. DiMagno MJ. Nitric oxide pathways and evidencebased perturbations in acute pancreatitis. Pancreatology 2007;7:403-8. doi:10.1159/000108956.

14. Banks PA, Freeman ML. Practice guidelines in acute pancreatitis. Am J Gastroenterol 2006;101:2379-400. doi: 10.1111/j.1572-0241.2006.00856.x.

15. Stevens T, Parsi MA, Walsh RM. Acute pancreatitis: Problems in adherence to guidelines. Cleve Clin J Med 2009;76:697-704. doi:10.3949/ccjm.76a.09060.

16. DiMagno MJ, Wamsteker E-J, Rai J, Rivera MA, Spaete JP, Ballard DD, et al. Do larger periprocedural fluid volumes reduce the severity of post- endoscopic retrograde cholangiopancreatography pancreatitis? Pancreas 2014;43:642-7. doi:10.1097/MPA.0000000000000101.

17. Buxbaum J, Yan A, Yeh K, Lane C, Nguyen N, Laine L. Aggressive hydration with lactated ringer's solution reduces pancreatitis After endoscopic retrograde cholangiopancreatography. Clin Gastroenterol Hepatol 2014;12:303-7 e1. doi:10.1016/j.cgh.2013.07.026.

18. Working Party of the British Society of Gastroenterology. Uk guidelines for the management of acute pancreatitis. Gut 2005;54 Suppl 3:iii1-9. doi:10.1136/gut.2004.057026.

19. Working Group IAP/APA Acute Pancreatitis Guidelines. Iap/apa evidence-based guidelines for the management of acute pancreatitis. Pancreatology 2013;13(4 Suppl 2):e1-15. doi:10.1016/j.pan.2013.07.063.

20. Muddana V, Whitcomb DC, Khalid A, Slivka A, Papachristou GI. Elevated serum creatinine as a marker of pancreatic necrosis in acute pancreatitis. Am J Gastroenterol 2009;104:164-70. doi:10.1038/ajg.2008.66.

21. GardnerTB, Olenec CA, Chertoff JD, Mackenzie TA, Robertson DJ. Hemoconcentration and pancreatic necrosis: Further defining the relationship. Pancreas 2006;33:16973. doi:10.1097/01.mpa.0000226885.32957.17.

22. Shaygan-Nejad A, Masjedizadeh AR, Ghavidel A, Ghojazadeh M, Khoshbaten M. Aggressive hydration with lactated ringer's solution as the prophylactic intervention for postendoscopic retrograde cholangiopancreatography pancreatitis: A randomized controlled doubleblind clinical trial. J Res Med Sci 2015;20:838-43. doi: 10.4103/1735-1995.170597. 